
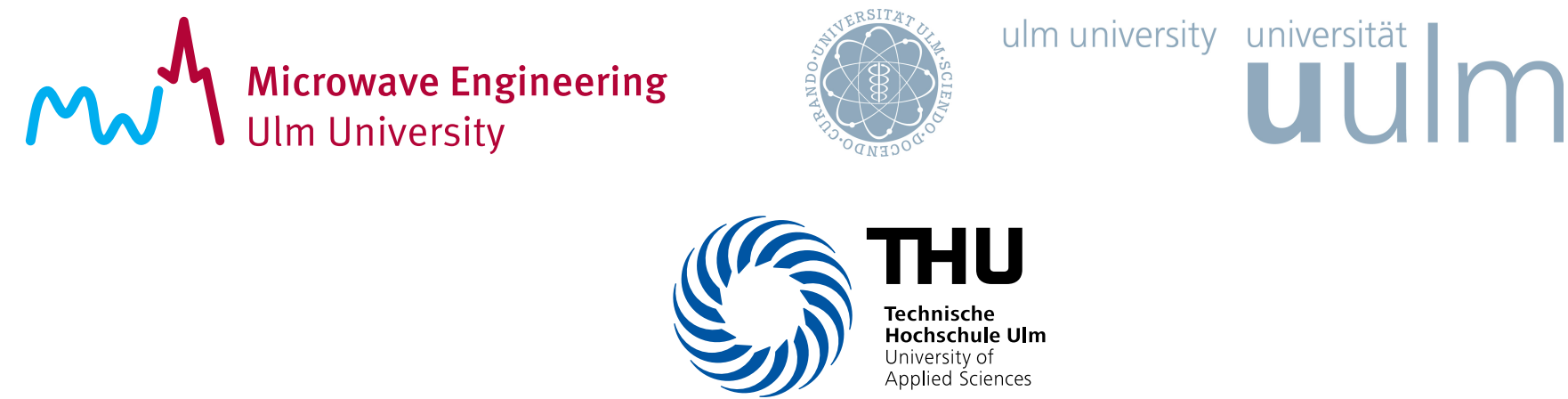

Design and Implementation of a FMCW GPR for UAV-based Mine Detection

Ralf Burr, Markus Schartel, Patrick Schmidt, Winfried Mayer, Thomas Walter, and Christian Waldschmidt

(C) 2018 IEEE. Personal use of this material is permitted. Permission from IEEE must be obtained for all other uses, in any current or future media, including reprinting/republishing this material for advertising or promotional purposes, creating new collective works, for resale or redistribution to servers or lists, or reuse of any copyrighted component of this work in other works. 


\title{
Design and Implementation of a FMCW GPR for UAV-based Mine Detection
}

\author{
Ralf Burr*, Markus Schartel ${ }^{\dagger}$, Patrick Schmidt*, Winfried Mayer ${ }^{\ddagger}$, Thomas Walter* and Christian Waldschmidt ${ }^{\dagger}$ \\ *Laboratory of Microtechnology, Ulm University of Applied Sciences, 89075 Ulm, Germany \\ ${ }^{\dagger}$ Institute of Microwave Engineering, Ulm University, 89081 Ulm, Germany \\ ${ }^{\ddagger}$ Endress+Hauser GmbH+Co. KG, 79689 Maulburg, Germany \\ Email: burr@hs-ulm.de
}

\begin{abstract}
Ground Penetrating Radar (GPR) is one of the tools supporting mine detection. In this contribution a wideband frequency modulated continuous wave (FMCW) GPR from $1 \mathrm{GHz}$ to $4 \mathrm{GHz}$ in a bistatic configuration is presented. This radar is designed so that it can be mounted on an unmanned aircraft vehicle (UAV). A compromise between weight, size, power consumption and penetration depth is found. The realization of the radar by means of frequency band splitting is presented. The merging of the two frequency bands is evaluated by measurements. The radar has been successfully integrated on a UAV and first measurements over a test field from the flight are presented.
\end{abstract}

\section{INTRODUCTION}

The military application of traps and, above all, the use of land mines has been widespread since the 13th century. These explosive weapons are buried a few centimeters below the ground and are triggered by the victim. However, the use of land mines is a permanent danger, as they pose a constant threat to the population for many decades after the military conflicts. Approximately 1 million people have lost their lives in the last 30 years through mines, with the number of unreported cases being much times higher. According to UN estimates, approximately 60 to 70 million mines are still active in more than 70 countries to date [1].

GPR is an essential tool for the detection of buried objects [2]. The detection is not only limited to metallic objects but also suitable for modern minimal metal mines [3]. State-ofthe-art GPR systems are either mounted on vehicles or used as hand-held devices. In both cases mine fields have to be entered for mine detection. To overcome this limitation, a broadband GPR sensor system that can be operated on an UAV has been developed.

This GPR sensor is carried by a low-altitude flying UAV. Measurements are recorded onboard and evaluated afterwards.

This paper is devided in 7 sections. Section II presents the system concept and the requirements for the GPR sensor. In section III the sensor concept and the broadband frequency synthesis is introduced. The manufactured sensor is presented in section IV. First characterization of the sensor with coaxilac cables as radar targets are presented in section $\mathrm{V}$ and section VI. Measurements with the GPR equipped UAV are shown in section VII. Section VIII gives a short conclusion.

\section{System CONCEPT AND REQUiREMENTS}

The usable GPR frequency range is primarily defined by the total path loss. The three main contributions are material loss, spreading loss and scattering loss [2]. The frequency dependence of the material loss is the most important parameter concerning ground penetration. In [4] and [5] frequencies up to maximum of $5 \mathrm{GHz}$ is proposed for the detection of land mines up to a maximum depth of $20 \mathrm{~cm}$. Due to the weight and size limitations on the UAV, the sensor underlies mechanical restrictions. The antenna size dominates mainly the whole sensor size. The size of the antenna scales with the lowest operating frequency $f_{u}$ of the antenna. The largest antenna dimension is approximately given by half wavelength $\lambda$ at the lowest radar frequency.

$$
\lambda_{\max }=\frac{c_{0}}{f_{u}}
$$

In order to keep the range resolution and penetration depth as high as possible and to keep the sensor for the UAV as small as possible, the used radar frequency range is set to $1 \mathrm{GHz}$ to $4 \mathrm{GHz}$.

In addition to the antenna, the battery affects the weight of the GPR sensor. Therefore, it is important to keep the power consumption of the entire system as low as possible to achieve sufficient measurement and flight times.

The basic principle of a radar is to measure the phase or time offset between transmitted and received signals. This means that the signal can be transmitted with one single antenna and received again with the same antenna. However, this requires directing components such as circulators. These components are either very heavy or not suitable for the required broad frequency range. Thus, in this case it is more practical to set up the radar in a bistatic configuration.

\section{SENSOR CONCEPT}

In the design phase of the sensor it was decided to use an FMCW radar. The main advantage of this modulation scheme is a higher mean power that can be radiated compared to a pulse radar [2]. The whole GPR sensor is divided into a main module and a receiver module. The corresponding block diagram is shown in Fig. 1. 


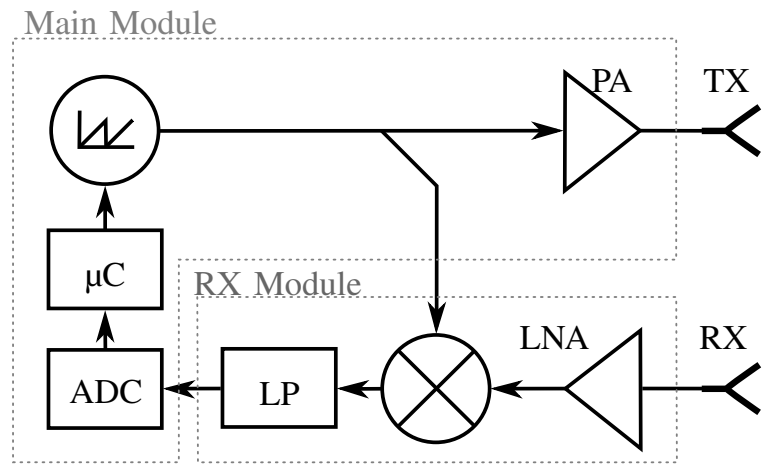

Fig. 1. Block diagram oft the whole GPR sensor.

\section{A. Main Module}

The main module consists out of four stacked printed circuit boards (PCBs). These boards are the radio frequency (RF) frontend, power supply board, micro-controller board, as well as the communication interface board for data logging.

In order to keep the power consumption low and to design the radar with commercially available components, a combination of fractional $\mathrm{N}$ phase locked loop (PLL) with integrated sweeper and voltage controlled oscillator (VCO) is used for the modulation. VCOs are only available for one frequency octave, whereby the entire frequency band is divided into two sub bands. In the following the frequency band $1 \mathrm{GHz}$ to $2 \mathrm{GHz}$ is named the low band and the $2 \mathrm{GHz}$ to $4 \mathrm{GHz}$ band is named the mid band. The schematic diagram of the frequency band division is shown in Fig. 2.

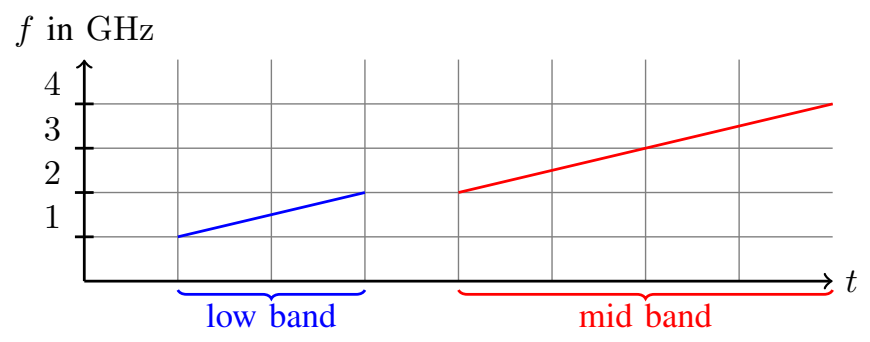

Fig. 2. Band splitting into low band and the mid band.

The key component of the RF frontend is the frequency synthesis. With a combination of a fractional N PLL and a VCO the low band frequency range $1 \mathrm{GHz}$ to $2 \mathrm{GHz}$ is covered. By a frequency doubler connected in series the low band frequency from $1 \mathrm{GHz}$ to $2 \mathrm{GHz}$ is doubled to the mid band frequency from $2 \mathrm{GHz}$ to $4 \mathrm{GHz}$. With additional switches, these two sub frequency bands are driven in time multiplex. The synthesized frequency sweep signal is split by means of a power splitter into the transmit signal (TX) and the local oscillator (LO) signal. The LO signal for the mixer is connected to the RX module via a coaxial cable. The block diagram of the RF frontend is shown in Fig. 3.

The PLL is configured with integer values as it is important that the slope of the low band corresponds to an integer even

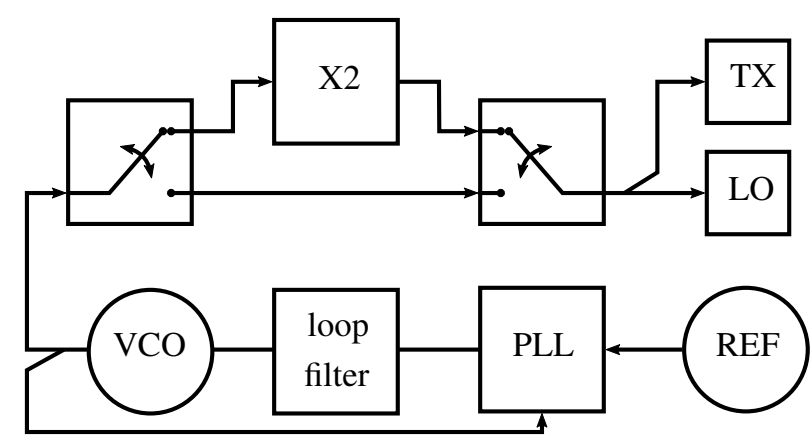

Fig. 3. Block diagram of the frequency synthesis using sub-band implementation.

number, otherwise the slope of the two bands can not be adjusted identically. In order to merge the intermediate frequency signal of the two frequency bands, the phase and amplitude must be calibrated at the transition point from the low band to the mid band. In order to give the IF filters a sufficient settling time, the frequency bands must overlap. Therefore the PLL has to be configured with a frequency overlap of a few $\mathrm{MHz}$ between the low and mid band. For a processing merging of the sub frequency bands a phase and amplitude matching is required. The exact phase matching is performed by the strict synchronization between frequency synthesis and analog-to-digital converter (ADC). The amplitude is adjustable by means of switchable intermediate frequency amplifiers and digital signal processing.

As micro-controller a DSP with integrated ADC with a maximal sample rate of $12 \mathrm{MHz}$ is used. This micro-controller also has 2 MBit of RAM to record several frequency sweeps.

\section{B. $R X$ Module}

The RX module is a separate PCB, which can be directly mounted at the receiving antenna. Due to the large antenna dimensions and the resulting distances, the receive path can be kept as short as possible. The RX module contains the signal mixing, receive signal amplification and intermediate frequency signal filtering. The receiver module is connected to the main module via two coaxial cables and one cable for control and power supplies.

\section{Final System Realization}

In Fig. 4 the stacked main module and the receiver module is shown. For the first test, a housing of the components was omitted. The dimensions of the main module is $80 \mathrm{~mm} \times 90 \mathrm{~mm} \times 50 \mathrm{~mm}$ and it has a weight of $125 \mathrm{~g}$. The board of the receiver module has a dimension of $45 \mathrm{~mm} \times 45 \mathrm{~mm} \times 10 \mathrm{~mm}$ and a weight of $20 \mathrm{~g}$. With the required cable set, the weight of the operative sensor is $195 \mathrm{~g}$. The power consumption in continuous operation is $4.8 \mathrm{~W}$. The input voltage must be in the range of $20 \mathrm{~V}$ to $30 \mathrm{~V}$. The measurement repetition frequency is $70 \mathrm{~Hz}$ and is mainly limited by the data interface. 


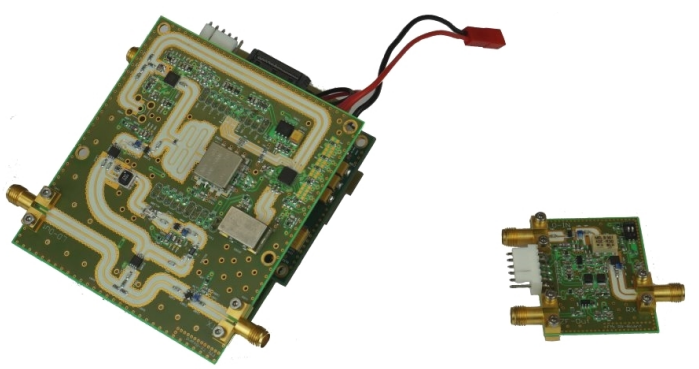

Fig. 4. Photo of the GPR sensor modules. On the left side the stacked main module and on the right side the receiver module

\section{LOW BAND EVALUATION}

In order check the linearity of the low band frequency sweep, the TX signal of the main module was recorded by an oscilloscope which has an analog bandwidth of $4 \mathrm{GHz}$. The signal transformed into the frequency domain is shown in Fig. 5. The frequency sweep from $1 \mathrm{GHz}$ to $2 \mathrm{GHz}$ in $256 \mu \mathrm{s}$ is clearly identifiable. The first harmonic from $2 \mathrm{GHz}$ to $4 \mathrm{GHz}$ is suppressed by $-20 \mathrm{~dB}$. By calculating the derivative of this frequency sweep, the error of the ramp linearity can be reduced to the resolution limit of the spectrogram.

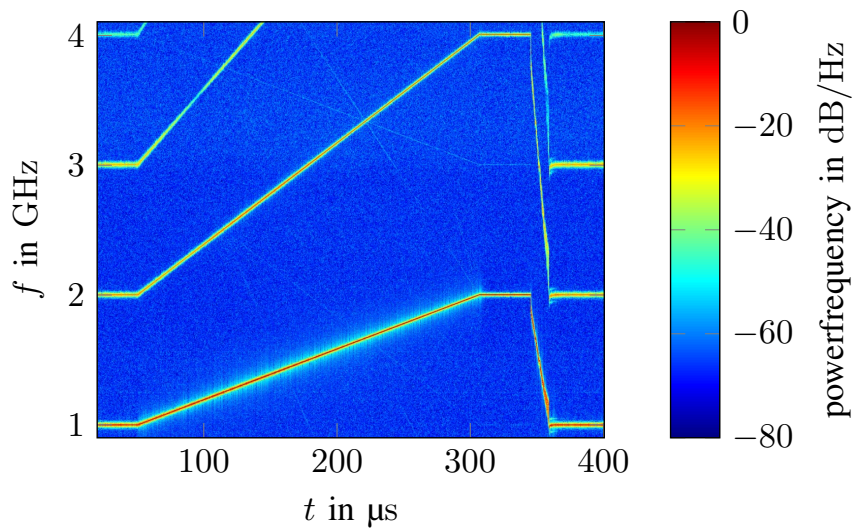

Fig. 5. Low band chirp, recorded with an oscilloscope and transformed in frequency domain.

\section{Vi. System Evaluation}

In order to analyze the function of the sensor the TX output of the main module is connected with the receiving input of the RX module via a delay line. In the middle of the delay line an open stitch line was added. The schematic diagram is shown in Fig. 6. This setup emulates two targets at distances of $6 \mathrm{~m}$ and $7.5 \mathrm{~m}$. The transmission signal at the TX port is attenuated by $80 \mathrm{~dB}$ simulating the free space round trip loss.

The recorded signal of each sub frequency band after windowing, six times zero padding and fourier transform is shown in Fig. 7. The red curve shows the spectrum of the low band with $1 \mathrm{GHz}$ bandwidth and the blue curve of the midband

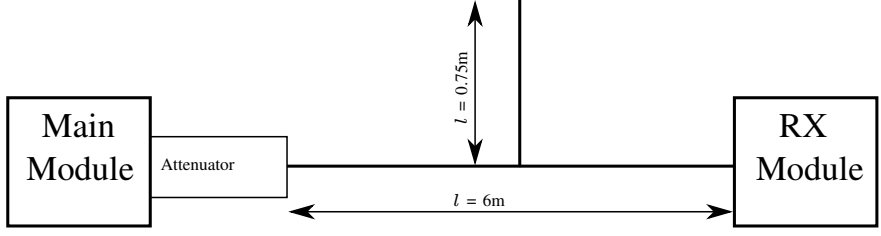

Fig. 6. Test setup with Target in $l=6 \mathrm{~m}$ and $l=7.5 \mathrm{~m}$.

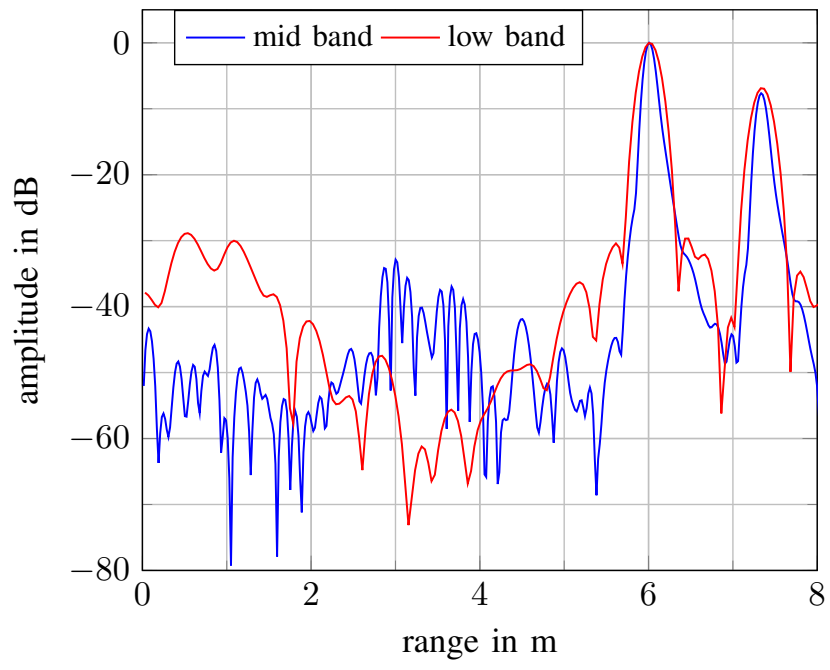

Fig. 7. Spectrum of the low and mid band.

with $2 \mathrm{GHz}$ bandwidth. The distances of the individual targets, each detected in both two bands, coincide well.

In Fig. 8 a comparison between the low band and the low band merged with the mid band is shown. To merge the subfrequency bands, their amplitudes must be adjusted to each other. Therefore the amplitude was determined by means of hilbert transform and corrected. In Fig. 8 can be seen that the target response becomes more narrow due to the higher bandwidth.

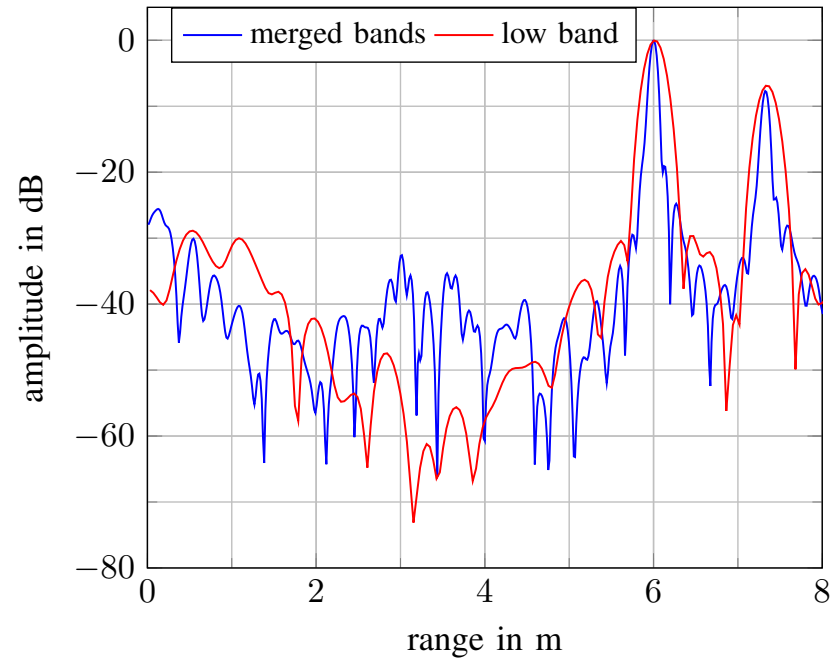

Fig. 8. Low band and merged low band with mid band. 
To check if the synchronization between the frequency synthesis and the ADC works, the stop phase of the respective target in the low band is compared to the start phase of the mid band. In order to determine the phase of a target more precisely, the resolution of a target in a given resolution cell is increased by means of a multiple approximated discrete fourier transform. The start and stop phases of the two targets are shown in Fig. 9. It can be seen that the stop phase varies from the start phase of each target by a few degrees.

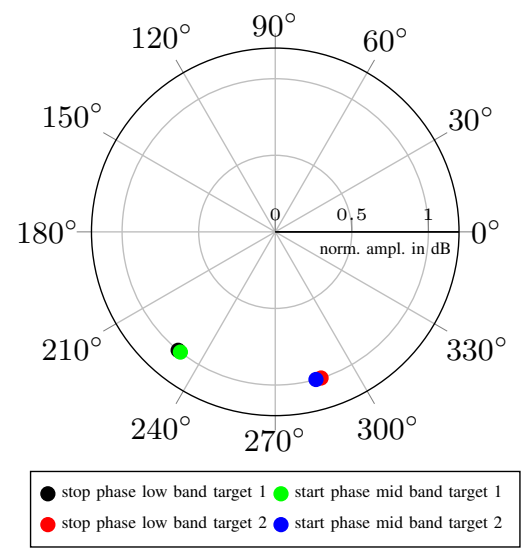

Fig. 9. Start and stop phase of target 1 and target 2

\section{SENSOR InTEgRation AND First Results}

A DJI Matrice M600 Pro is used as flight platform for the GPR sensor. In addition to the GPR sensor, a single board computer for data recording and a lidar for measuring the flight altitude above ground are used. The whole measuring system with two horn antennas is shown in Fig. 10.

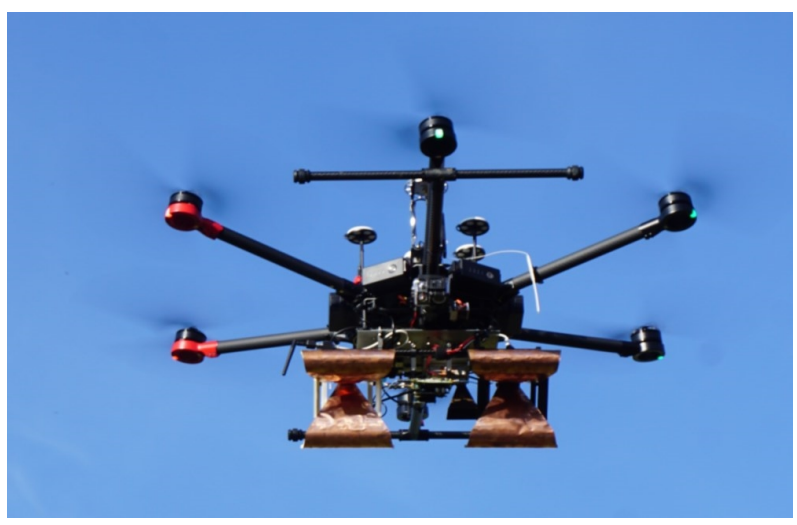

Fig. 10. Photo of the carrier platform DJI M600 Pro equipped with the GPR sensor.

A test field was equipped with two different sized corner reflectors and several filled plastic cans as shown in Fig. 11. The flight was controlled by hand and follows along the reflectors.

The range compressed radar data measurements are shown in Fig. 12. A strong crosstalk between the antennas up to a range of $2 \mathrm{~m}$ can be seen. The first reflections from the ground

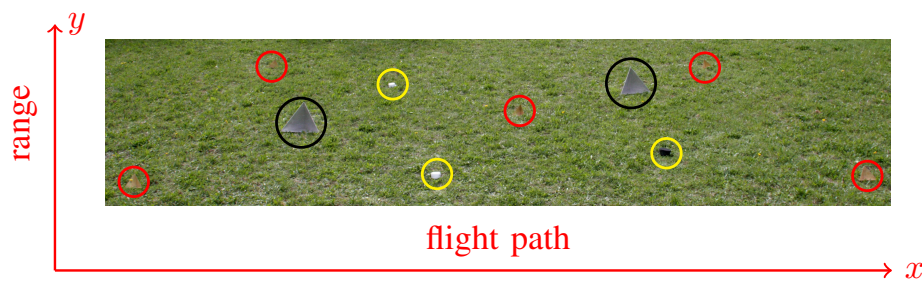

Fig. 11. Photo of the setup from the perspective of the radar.

are in a range of approximately $2.5 \mathrm{~m}$. Also the hyperbolas, caused by different distances during the passing flight, can be recognized. The distorted shapes of the hyperbolas are caused by the non-straight flight trajectory.

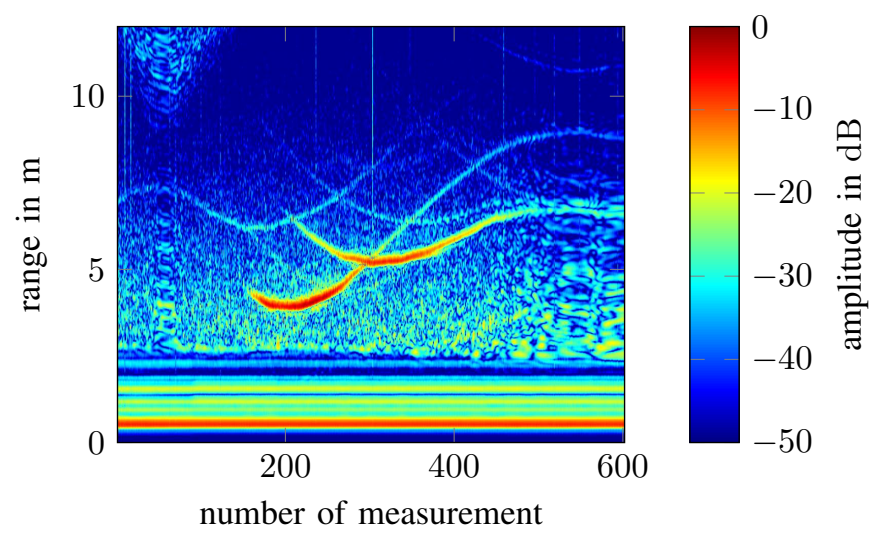

Fig. 12. Range compressed radar measurement over test field.

\section{CONCLUSION}

A GPR with sub band realization has been presented. The single band performance and sub band merging was verified by a cable measurement. The GPR sensor partitioning was done with the splitting of the sensor into a main module and a receiver module. With a power consumption of $4.8 \mathrm{~W}$ and a weight of less than $200 \mathrm{~g}$, it is well suited for UAV platforms. Finally, the radar was integrated with horn antennas on a UAV and measurements were taken over a field with corner reflectors as targets.

\section{ACKNOWLEDGMENT}

The authors thank the Urs Endress foundation for supporting this work (https://www.ue-stiftung.org).

\section{REFERENCES}

[1] The International Campaign to Ban Landmines, "Landmine monitor 2016," Monitoring and Research Committee, ICBL-CMC Governance Board, November 2016.

[2] D. Daniels, Ground Penetrating Radar, ser. Electromagnetics and Radar Series. Institution of Engineering and Technology, 2004, no. Bd. 1.

[3] B. Scheers, M. Piette, and A. V. Vorst, "The detection of ap mines using uwb gpr," in 1998 Second International Conference on the Detection of Abandoned Land Mines (IEE Conf. Publ. No. 458), Oct 1998, pp. 50-54.

[4] C. Fischer and W. Wiesbeck, "Multistatic gpr for antipersonnel mine detection," in IGARSS 2001, vol. 6, 2001, pp. 2721-2723 vol.6.

[5] M. Fritzsche, Anwendungen von Verfahren der Mustererkennung zur Detektion von Landminen mit Georadaren. Universitt Karlsruhe, 2001. 